\title{
Late neurodevelopmental outcome after repair of total anomalous pulmonary venous connection
}

\author{
Paul M. Kirshbom, MD, ${ }^{a}$ Thomas B. Flynn, PhD, ${ }^{d}$ Robert R. Clancy, MD, ${ }^{e}$ Richard F. Ittenbach, PhD, ${ }^{f}$ \\ Diane M. Hartman, RN, ${ }^{\mathrm{b}}$ Stephen M. Paridon, MD, ${ }^{\mathrm{c}}$ Gil Wernovsky, MD, ${ }^{\mathrm{c}}$ Thomas L. Spray, MD, ${ }^{\mathrm{b}}$ and \\ J. William Gaynor, MD ${ }^{\mathrm{b}}$
}

From the Division of Cardiothoracic Surgery, ${ }^{\mathrm{a}}$ Emory University, Atlanta, Ga, and the Divisions of Cardiothoracic Surgery, ${ }^{\mathrm{b}}$ Cardiology, ${ }^{\mathrm{c}}$ Psychology, ${ }^{\mathrm{d}}$ Neurology, ${ }^{\mathrm{e}}$ and Biostatistics and Epidemiology, ${ }^{\mathrm{f}}$ The Children's Hospital of Philadelphia, Philadelphia, Pa.

Supported in part by a grant from the Fannie E. Ripple Foundation and the National Institutes of Health General Clinical Research Center (grant no. M01-RR-00240).

Received for publication June 8, 2004; revisions received Aug 10, 2004; accepted for publication Aug 16, 2004.

Address for reprints: Paul M. Kirshbom, MD, 1365 Clifton Rd, Suite A2100, Cardiothoracic Surgery, Emory Clinic Bldg. A, Atlanta, GA 30322 (E-mail: paul_kirshbom@ emoryhealthcare.org).

J Thorac Cardiovasc Surg 2005;129:1091-7 $0022-5223 / \$ 30.00$

Copyright (c) 2005 by The American Association for Thoracic Surgery

doi:10.1016/j.jtcvs.2004.08.013
Objective: We sought to define the neurodevelopmental status of school-aged survivors of total anomalous pulmonary venous connection repaired during infancy.

Methods: All school-aged survivors of total anomalous pulmonary venous connection repair performed at a single institution were eligible. Thirty children returned for neurologic examination and neurodevelopmental testing.

Results: The median age at total anomalous pulmonary venous connection repair was 16 days (range, 1-141 days), and age at testing was 11 years (range, 6-19 years). Pulmonary venous return was supracardiac in 14 patients, infracardiac in 12 patients, cardiac in 3 patients, and mixed in 1 patient. Preoperative obstructed total anomalous pulmonary venous connection was present in 6 patients. Circulatory arrest was used in all repairs, with a median duration of 35 minutes (range, 17-55 minutes). At follow-up, microcephaly (head circumference $<5 \%$ ) was present in $28 \%$. Neuromuscular examination was suspect or abnormal in $27 \%$. Mean Full-scale IQ $(95.3 \pm 18.5)$ and Verbal IQ $(98.6 \pm 20.2)$ were not different from population norms, but Performance IQ $(92.3 \pm 16.9)$ was significantly lower than population norms $(P=.02)$. Fine motor skills and visual-motor coordination were significantly impaired $(P<.01$ for Grooved Pegboard and Test of Visual-Motor Integration). Patients with total anomalous pulmonary venous connection also had difficulty with tests of attention (Test of Everyday Attention for Children, $P<.01$ ), but results of tests of memory function were not significantly different from population norms.

Conclusions: School-aged survivors of infant total anomalous pulmonary venous connection repair exhibit a significant incidence of neurodevelopmental difficulties. Fine motor function, visual-motor integration, and attention are the most commonly affected domains. Evaluation of these children is indicated to identify those who are at risk for learning disabilities and who could benefit from early intervention.

$\mathrm{E}$ arly outcomes after surgical repair of total anomalous pulmonary venous connection (TAPVC) have steadily improved over the past decades,,${ }^{1-4}$ but late outcomes have not been well characterized. Although studies have reported excellent long-term survival and functional status for patients with TAPVC, there is little information available concerning late neuropsychologic outcome. ${ }^{1,5}$ Over the past several years, there has been an increasing recognition of late neurodevelopmental deficits in school-aged survivors of neonatal cardiac surgery. ${ }^{6-8}$

We recently published a study based on parental perceptions of children who underwent TAPVC repair as infants. ${ }^{9}$ Several factors, including preoperative pulmonary venous obstruction and the presence of genetic syndromes, were associated with worse overall health and school performance as evaluated by parents in 
TABLE 1. Demographics and anatomic data $(n=30)$

\begin{tabular}{lc}
\hline Demographics & \\
Age at the time of the operation (d) & $35 \pm 43$ \\
Birth weight (g) & $3290 \pm 712$ \\
Gestational age (wk) & $39 \pm 2$ \\
Male/female sex & $21 / 9$ \\
Anatomy & \\
Supracardiac, $\mathrm{n}(\%)$ & $14(47)$ \\
Cardiac, $\mathrm{n}(\%)$ & $3(10)$ \\
Infracardiac, $\mathrm{n}(\%)$ & $12(40)$ \\
Mixed, $\mathrm{n}(\%)$ & $1(3)$ \\
Pulmonary venous obstruction, $\mathrm{n}(\%)$ & $6(20)$ \\
\hline
\end{tabular}

response to a telephone survey. These results suggested the need for a more direct and detailed evaluation of this patient population to better define their long-term outcomes and to help identify subgroups at greater risk for neuropsychologic dysfunction and diminished quality of life. Hence the primary goal of this study was to characterize the neuropsychologic function of school-aged survivors of TAPVC repair. The secondary goal was to determine whether available preoperative or intraoperative variables significantly influenced late neuropsychologic status.

\section{Methods}

\section{Patient Population}

The pool of potential subjects eligible to participate in this study included all patients with simple TAPVC admitted to The Children's Hospital of Philadelphia between January 1, 1983, and January 31 , 2001. Simple TAPVC was defined as TAPVC with no other cardiac anomalies except for patent foramen ovale, atrial septal defect, aortic coarctation, or patent ductus arteriosus. ${ }^{9}$ A total pool of 104 children were identified, 4 of whom died before surgical intervention. Of the 100 children who survived to the operation, 84 were alive at last follow-up. Of these 84 late survivors, 60 were 6 years of age or older at the time of this study. The parents-guardians of 42 of these 60 children were successfully contacted. Of these 42 families, 30 consented to participate in this study and were enrolled.

The enrolled subjects had undergone surgical repair between November 1983 and November 1996. These patients returned to The Children's Hospital of Philadelphia for a day-long series of assessments, including neuropsychologic testing, echocardiography, and neurologic examination, between June 2002 and January 2003. The surgical management and anatomic details for the group as a whole have been previously described. ${ }^{9}$ This study was approved by The Children's Hospital of Philadelphia Institutional Review Board, Committee for Protection of Human Subjects.

\section{Neuropsychologic Testing}

Cognitive ability was assessed by using the Wechsler Abbreviated Scale of Intelligence providing Verbal, Performance, and Fullscale IQ scores. Academic achievement was assessed with selected subtests from the Wechsler Individual Achievement Test, Second
TABLE 2. Intraoperative and postoperative data

\begin{tabular}{lc}
\hline Operative data & \\
Intubated preoperatively (n) & 10 \\
Preoperative ECMO support (n) & 1 \\
Bypass time (min) & $71 \pm 14$ \\
Circulatory arrest (min) & $35 \pm 10$ \\
Postoperative data & \\
ICU length of stay (d) & $8 \pm 11$ \\
Length of stay, operation to discharge (d) & $13 \pm 12$ \\
\hline ECMO, Extracorporeal membrane oxygenation; ICU, intensive care unit.
\end{tabular}

Edition (WIAT-II). Basic reading was assessed on the basis of measures of single-word reading and novel word decoding (WIAT-II Word Reading and Pseudoword Decoding), written language on the basis of the WIAT-II Spelling subtest, and math calculations on the basis of the WIAT-II Numerical Operations subtest. Neuropsychologic testing also included measures of attention (Test of Everyday Attention for Children [TEA-Ch], Sky Search, Score!, Creature Counting, and Sky Search Dual Task subtests), executive function (Behavior Rating Inventory of Executive Function, Parent Form; Controlled Oral Word Association), memory (Children's Memory Scale [CMS], core subtests), speech comprehension (Clinical Evaluation of Language FundamentalsThird Edition, Concepts and Directions), and fine motor (Grooved Pegboard) and visual-motor skills (Beery-Buctenica Developmental Test of Visual-Motor Integration-Fourth Edition, Revised; NEPSY Visuomotor Precision). Parent-report measures of their child's behavior and personality (Behavior Assessment System for Children [BASC]) were also administered.

\section{Neurologic Examination}

A pediatric neurologist examined all of the children. The examination included head circumference, cranial nerve function, extremity function, tone, and reflexes. Summary scores for general neuromuscular and overall neurologic status were graded as normal, suspect, or abnormal.

\section{Statistical Analysis}

Data analysis occurred in 4 phases. Phase I consisted of generating descriptive measures of central tendency, variability, and association for all relevant variables in the dataset. Frequency tables, box plots, and histograms were used to complement the summary statistics for continuous, as well as categorical, variables. Phase II consisted of 13 different 1-sample $t$ tests in which the scores of children in our sample were compared with population norms for the 13 standardized measures listed previously. Collectively, all 13 tests represented 5 domains of interest (intelligence, academic achievement, attention, memory, and motor control and visualmotor integration). Phase III consisted of classifying and tabulating the number of children deemed to be at risk in one or more of the following clinical domains (cognition, academic achievement, attention, and visual-motor integration). A child was classified as at risk for impairment if she or he scored between 1 and 2 SDs less than the mean when compared with age norms on a given instrument. A child was classified as impaired if she or he scored 2 or 
TABLE 3. Developmental test scores

\begin{tabular}{|c|c|c|c|}
\hline Test & Patients with TAPVC & Population norms & $P$ value \\
\hline \multicolumn{4}{|l|}{ Intelligence } \\
\hline \multicolumn{4}{|l|}{ WASI } \\
\hline Full-scale IO & $95.3 \pm 18.5$ & $100 \pm 15$ & .19 \\
\hline Performance 10 & $92.3 \pm 16.9$ & $100 \pm 15$ & .02 \\
\hline Verbal IO & $98.6 \pm 20.2$ & $100 \pm 15$ & .72 \\
\hline \multicolumn{4}{|l|}{ Academic achievement } \\
\hline \multicolumn{4}{|l|}{ WIAT-II } \\
\hline Word Reading & $97.6 \pm 21.9$ & $100 \pm 15$ & .55 \\
\hline Numerical Operations & $88.2 \pm 16.5$ & $100 \pm 15$ & $<.01$ \\
\hline \multicolumn{4}{|l|}{ Attention } \\
\hline \multicolumn{4}{|l|}{ CMS } \\
\hline Attention/Concentration & $95.1 \pm 18.7$ & $100 \pm 15$ & .20 \\
\hline \multicolumn{4}{|l|}{ Index } \\
\hline \multicolumn{4}{|l|}{ BASC } \\
\hline Attention & $52.0 \pm 11.4$ & $50 \pm 10$ & .37 \\
\hline \multicolumn{4}{|l|}{ TEA-Ch } \\
\hline Score! & $7.7 \pm 3.7$ & $10 \pm 3$ & $<.01$ \\
\hline Sky Search Dual Task & $5.4 \pm 3.1$ & $10 \pm 3$ & $<.01$ \\
\hline \multicolumn{4}{|l|}{ Memory } \\
\hline \multicolumn{4}{|l|}{ CMS } \\
\hline General Memory Index & $102.1 \pm 23.9$ & $100 \pm 15$ & .65 \\
\hline \multicolumn{4}{|l|}{ Motor and Visual-Motor } \\
\hline \multicolumn{4}{|l|}{ Functions } \\
\hline \multicolumn{4}{|l|}{ Grooved Pegboard } \\
\hline Dominant hand & $-1.9 \pm 2.3(z$ score $)$ & $0 \pm 1$ & $<.01$ \\
\hline VMI & $89.5 \pm 16.0$ & $100 \pm 15$ & $<.01$ \\
\hline NEPSY-Visuomotor Precision & $8.4 \pm 3.5$ & $10 \pm 3$ & .04 \\
\hline
\end{tabular}

TAPVC, Total anomalous pulmonary venous connection; WASI, Wechsler Abbreviated Scale of Intelligence; WIAT-II, Wechsler Individual Achievement Test-Second Edition; CMS, Children's Memory Scale; BASC, Behavior Assessment System for Children, Parent Rating Scales; TEA-Ch, Test of Everyday Attention for Children; VMI, Beery-Buktenica Developmental Test of Visual-Motor Integration, Fourth Edition (Revised); NEPSY, Visual-motor precision.

more SDs less than the mean. Finally, in Phase IV single-covariate and multiple-covariate risk-factor prediction equations were generated and tested for statistical significance by using the 13 previously mentioned scaled scores as outcomes and 21 different potential risk factors as covariates. Model selection was conducted sequentially, first by identifying all single-covariate risk factors likely to contribute to a multiple-covariate model (ie, $P<.10$ ) by either simple multiple regression or simple logistic regression, depending on the nature of the end point. Multiple-covariate models were then chosen for each of the 13 outcomes by using Wald statistics, conditional error tests, likelihood ratio tests, $R^{2}$ values, or a combination of these methods in addition to relevant clinical and theoretic considerations. Hypothesis-wise error rates for the 1-sample tests were held constant at the .005 level by Tukey, Ciminera, and Heyse adjustment for multiple related comparisons. ${ }^{10}$ Because of the exploratory nature of the study and the general lack of available information on similar models in the literature, $\alpha$ values were not adjusted for the inferential models. All data were analyzed by SAS Version 9.0 software (SAS Institute, Inc, Cary, NC).

\section{Results}

Patient demographics and anatomy of the pulmonary venous drainage are summarized in Table 1 . The median age of the children at testing was 11.1 years (range, 5.8-18.7 years). Their median weight was $38 \mathrm{~kg}$ (range, 16-85 kg), and height was $144 \mathrm{~cm}$ (range, 104-171 cm). This was a relatively healthy group, with none of the children taking routine cardiac medications.

Echocardiograms were essentially normal in 29 of the 30 children. No child had greater than mild tricuspid regurgitation, and the right ventricular pressure estimate based on the tricuspid regurgitation jet measured in 18 patients was $21 \pm 4 \mathrm{~mm} \mathrm{Hg}$. There was evidence of mild flow acceleration at the pulmonary venous anastomosis in one child who was asymptomatic and whose right ventricular pressure estimate was $28 \mathrm{~mm} \mathrm{Hg}$.

Most of the participating families were middle class, with a median Hollingshead occupational score of 4 (range, 
TABLE 4. Summary of neurodevelopmental outcomes compared with population norms

\begin{tabular}{|c|c|c|c|}
\hline & Normal (\%) & At risk (\%) & Impaired (\%) \\
\hline \multicolumn{4}{|l|}{ Intelligence (WASI) } \\
\hline Full-scale IO ( $\mathrm{n}=28)$ & 71.4 & 17.8 & 10.7 \\
\hline \multicolumn{4}{|l|}{ Academic achievement (WIAT) } \\
\hline Word Reading $(\mathrm{n}=29)$ & 75.9 & 13.8 & 10.3 \\
\hline Numerical Operations $(\mathrm{n}=29$ ) & 69 & 17.2 & 13.8 \\
\hline \multicolumn{4}{|l|}{ Attention (TEA-Ch) } \\
\hline Score! $(n=25)$ & 56 & 36 & 8 \\
\hline Sky Search Dual Task (n = 24) & 41.7 & 29.2 & 29.2 \\
\hline \multicolumn{4}{|l|}{ Memory (CMS) } \\
\hline General Memory Index ( $\mathrm{n}=26$ ) & 84.6 & 3.8 & 11.5 \\
\hline \multicolumn{4}{|l|}{ Motor and Visual-Motor Functions } \\
\hline Grooved Pegboard ( $n=28$ ) & 39.3 & 25 & 35.7 \\
\hline VMI $(\mathrm{n}=28)$ & 57.1 & 32.1 & 10.7 \\
\hline NEPSY (n = 24) & 66.7 & 25 & 8.3 \\
\hline
\end{tabular}

Normal, More than -1 SD; at risk, between -1 SD and -2 SD; impaired, less than -2 SD; WASI, Wechsler Abbreviated Scale of Intelligence; WIAT-II, Wechsler Individual Achievement Test-Second Edition; TEA-Ch, Test of Everyday Attention for Children; CMS, Children's Memory Scale; VMI, BeeryBuktenica Developmental Test of Visual-Motor Integration, Fourth Edition (Revised); NEPSY, Visual-motor precision.

TABLE 5. Neurologic examination results

\begin{tabular}{lccrr}
\hline & Hyperactivity & Inattentiveness & $\begin{array}{c}\text { Neuromuscular } \\
\text { assessment }\end{array}$ & $\begin{array}{c}\text { Overall neurologic } \\
\text { assessment }\end{array}$ \\
\hline Normal & $22(73 \%)$ & $16(53 \%)$ & $23(77 \%)$ & $18(60 \%)$ \\
Suspect & & $4(13 \%)$ & $5(17 \%)$ \\
Abnormal & $8(27 \%)$ & $14(47 \%)$ & $3(10 \%)$ & $7(23 \%)$ \\
\hline
\end{tabular}

1-5). Ten (33\%) of the mothers completed high school, and an additional $19(63 \%)$ attended or completed post-high school education.

Perioperative data are summarized in Table 2. All patients had a period of deep hypothermic circulatory arrest during their procedure, ranging from 17 to 55 minutes in duration. The mean postoperative intensive care unit length of stay was 8 days. Patients who required preoperative intubation $(n=10)$ were significantly younger than those who were not intubated ( $6 \pm 6$ days vs $47 \pm 46$ days, $P=.009$ ), and they required significantly longer postoperative intensive care unit stays (16 \pm 17 days vs $4 \pm 4$ days, $P=.005$ ). Patients with evidence of preoperative pulmonary venous obstruction $(n=6)$ were more likely to require preoperative intubation (5/6 [83\%] vs $5 / 24$ [21\%], $P=.003)$.

A comparison between the study patients who enrolled and those patients who were eligible but did not return demonstrated no significant differences between the groups for the demographic and perioperative variables listed in Tables 1 and 2 .

\section{Developmental Testing}

Intelligence and academic achievement. The study group and population norms for the Wechsler Abbreviated
Scale of Intelligence (Full-scale IQ, Verbal IQ, and Performance IQ) and WIAT-II (Numerical Operations and Word Reading subtests) are included in Tables 3 and 4. The Full-scale and Verbal IQ scores of patients with TAPVC were not significantly different from the population norms, but their Performance IQ scores were significantly lower at $92 \pm 17$ versus $100 \pm 15(P=.02)$. Similarly, the patients' scores on a measure of single-word reading (WIAT-II Word Reading) were comparable with population norms, whereas their performance on a measure of math calculations (WIAT-II Numerical Operations) was significantly lower $(P<.01)$.

Memory and attention. The results of the Memory and Attention subtests from the CMS, TEA-Ch, and BASC are also included in Tables 3 and 4 . The patients with TAPVC were not significantly different from population norms on a broad measure of general memory functioning. Regarding attention, parental assessment of the patients' attention on the BASC was within the normal range, as was the CMS Attention/Concentration Index score. However, on direct testing of sustained attention (TEA-Ch Score! Subtest) and divided attention (TEA-Ch Sky Search Dual Task), patients with TAPVC obtained significantly lower than average scores on both tests $(P<.01)$. 


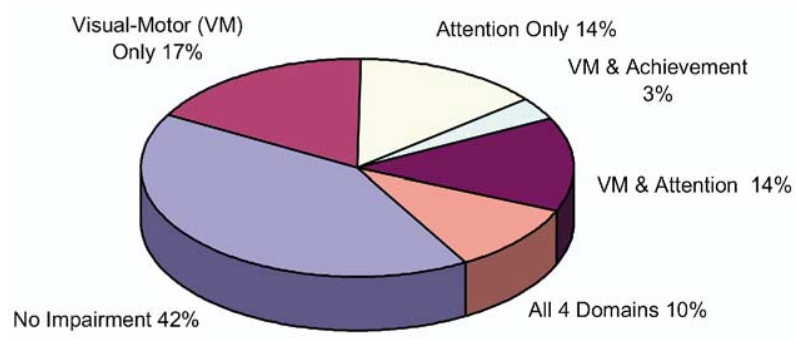

Figure 1. Percentage of children with significant dysfunction in each of the domains of neurodevelopmental function tested. Impairment was defined as greater than 2 SDs below the mean for population norms on the following tests: cognition, Full-scale I0; achievement, WIAT-II Numerical Operations or Word Reading subtests; attention, TEA-Ch Score!, TEA-Ch Sky Search Dual Task subtest, CMS Attention/Concentration Index; motor/visual-motor (VM), VMI, NEPSY Visuomotor Precision subtest, or Grooved Pegboard Test.

Motor function. As a group, patients with TAPVC scored significantly lower than population norms on all administered tests of fine motor function and visual-motor integration. Results of the Beery-Buktenica Developmental Test of Visual-Motor Integration, Fourth Edition, Revised (VMI); NEPSY; and Grooved Pegboard are shown in Tables 3 and 4 .

\section{Neurologic Examination}

Neurologic function of 7 (23\%) of the 30 children was rated as abnormal and that of $5(17 \%)$ was rated as "suspect" on the overall neurologic assessment (Table 5). Abnormalities in fine motor control and dexterity were the reasons for these assessments in the majority of these patients. Microcephaly (head circumference $\leq 5$ th percentile) was present in $8(28 \%)$ of the 29 children.

\section{Perioperative Factors Associated with Late Neurodevelopmental Outcomes}

Twenty-one potential perioperative risk factors were tested for inclusion in the multivariable model predicting late neurodevelopmental test results. Potential risk factors included preoperative factors (gestational age, birth weight, sex, preoperative cardiac arrest, intubation, mechanical circulatory support, chromosomal syndromes, pulmonary venous drainage anatomy, pulmonary venous obstruction, and familial Hollingshead socioeconomic score), operative factors (age and weight at the time of the operation, date of the operation, emergency operation, cardiopulmonary bypass and circulatory arrest duration, and use of an atrial septal defect patch), and postoperative factors (hospital length of stay, postoperative seizures, postoperative pulmonary venous obstruction, and need for late reintervention). Higher gestational age significantly improved Full-scale and Verbal IQ scores with a nonadjusted $\alpha$ value $(P=.04$ and .03 , respectively). The effect of circulatory arrest duration on outcomes was inconsistent. Increasing circulatory arrest duration negatively affected WIAT-II Numerical Operations $(P=.04)$ and CMS General Memory $(P<.01)$ scores, but increasing circulatory arrest duration also improved VMI scores $(P<.01)$. Use of a patch to close the atrial septal defect also had an inconsistent but frequently significant effect on several outcome variables. The use of an atrial septal defect patch had a positive effect on Full-scale IQ $(P=$ $.05)$, Verbal IQ $(P=.05)$, and CMS General Memory $(P=$ $.02)$ scores, whereas patch use negatively affected TEA-Ch Sky Search Dual Task $(P=.03)$ and VMI $(P=.01)$ scores. Grooved Pegboard results with the dominant hand were positively affected by more recent date of surgical intervention $(P$ $<.01)$ and weight at admission $(P=.02)$. There were no significant predictors of Performance IQ, WIAT-II Word Reading, TEA-Ch Score!, CMS Attention/Concentration, BASC Attention, or NEPSY scores.

\section{Discussion}

Earlier studies have reviewed short-term and long-term outcomes after repair of TAPVC. ${ }^{1-3,5,11}$ These studies have demonstrated steadily improving hospital survival over the last 20 years, with most reporting greater than $90 \%$ survival to discharge and approximately $80 \%$ to $85 \%$ long-term survival. Despite these results, relatively little detailed information has been published regarding long-term functional status. Bando and colleagues ${ }^{1}$ reported that $98 \%$ of operative survivors were alive at late follow-up and in New York Heart Association (NYHA) class I. In an evaluation of 44 patients with TAPVC repaired between 1973 and 1998, Bogers and associates ${ }^{5}$ reported an $84 \%$ actuarial survival at 15 years, with all of the patients at an age-appropriate point in school or employed. In contrast to these relatively optimistic reports is the consistent pattern of neurodevelopmental dysfunction demonstrated in children who underwent neonatal repair of transposition of the great arteries, as reported in the Boston Circulatory Arrest Study ${ }^{6,7}$ and by others. ${ }^{8}$ Because subgroups of patients with TAPVC and transposition experience similar risk factors for long-term neurodevelopmental dysfunction, including early hemodynamic instability, prolonged postoperative intensive care unit stay, and the use of circulatory arrest during surgical repair, one might expect their long-term neurodevelopmental outcomes to be similar. One of the primary goals of the current study was to reconcile this apparent incongruity.

As previously reported, the current study population is comparable with other reported series of patients with TAPVC. ${ }^{9}$ The overall hospital mortality for the group as a whole was $14 \%$, and the 15 -year actuarial survival was $84 \%$. All infants underwent deep hypothermic circulatory 
arrest with a mean duration of 35 minutes. Thirteen of the original 100 patients required reintervention for pulmonary venous obstruction, with 3 of these procedures performed during the original hospitalization. A parental questionnaire-based evaluation suggested that these patients were doing quite well, with $91 \%$ of the parents assessing their child's overall health as "excellent" or "good," and 69\% viewing their school performance as "above average" or "average." Despite these promising preliminary results, neuropsychologic testing of long-term survivors in the current study demonstrated a pattern of dysfunction quite similar to that found among long-term survivors of the arterial switch procedure for transposition of the great arteries.

Hovels-Gurich and coworkers ${ }^{8}$ reported that $55 \%$ of their patients with transposition were impaired in one or more domains of function (neurologic, motor, intelligence-ability, or speech). In the current study we found that $58 \%$ of the patients with TAPVC studied were impaired in one or more of the following domains: intelligence, academic achievement, attention, or motor function (Figure 1). Although the domains of function tested and the specific tests used were different, the overall findings were comparable. Meanwhile, in the 8-year follow-up of the Boston Circulatory Arrest Trial, Bellinger and associates ${ }^{7}$ described a "...behavioral signature for children after surgery to repair D-TGA." This signature or pattern of dysfunction was characterized by deficits in "motor function and visual-spatial skills," which is strikingly similar to the findings in our TAPVC study group. The data from these reports and others support the concern that infants born with cardiac defects associated with hemodynamic instability during the neonatal period and who require neonatal cardiac repair are at risk for late neurodevelopmental dysfunction. Even children whose repair leaves them with normal hemodynamics and no further need for cardiovascular intervention clearly fall into this group.

Bellinger and associates ${ }^{7}$ also found that their patients with transposition had difficulty with higher-order executive function, including working memory and sustained attention. Our evaluation of the ability of patients with TAPVC to focus on tasks and attentiveness also yielded interesting results. Although these children scored within the normal range on basic tests of attention, they scored significantly below population norms on more challenging tests requiring sustained attention over time or the division of attention between 2 tasks. Meanwhile, parental evaluation of their child's attention fell within the normal range, suggesting that although they were able to compensate to some degree, these children's needs might not be fully recognized and appreciated.

Multiple covariate analysis suggested several risk factors associated with late neurodevelopmental dysfunction. Decreasing gestational age was an independent risk factor for lower Full-scale and Verbal IQ, and a more recent date of operation and higher weight at admission were associated with better Grooved Pegboard performance. Circulatory arrest duration and the use of an atrial septal defect patch both had inconsistent effects on several variables that were associated with better outcomes on some test measures and worse outcomes for others.

There are several potential limitations to this study. First, these patients had operations over a 13-year period, during which time surgical and intensive care techniques evolved considerably. Although the date of the operation was included in the multivariable analysis, it is difficult to account for the many changes that might have taken place over this time span. Second, only $50 \%$ of the patients eligible to return for the study were successfully contacted and agreed to return. Although a comparison between the returnees and nonreturnees using all of the variables entered into the multivariable model did not demonstrate any significant differences between the groups, the potential for a selection bias certainly exists. Also, complete data were only available for 29 patients, which is a relatively small sample size. Results likely would have been more stable in larger samples.

In conclusion, the long-term outcome for patients with TAPVC repaired in infancy is quite good. The majority of these patients are at an age-appropriate point in schooling, and their overall health is good. However, as in other patient groups with congenital heart defects necessitating surgical repair in infancy, children with a history of TAPVC show a pattern of neurodevelopmental dysfunction characterized by an increased proportion of abnormal findings on neurologic examination, impaired fine motor speed and control, lower Performance IQ, and difficulties with demanding attentional tasks. Unrecognized and inadequately met educational needs might be a source of frustration for children with a history of TAPVC repair. Some might benefit from early intervention to assist them with learning and attention disorders. Neuropsychologic evaluation of patients with TAPVC operated on during infancy is warranted to identify long-term neurocognitive deficits.

\section{References}

1. Bando K, Turrentine M, Ensing G, et al. Surgical management of total anomalous pulmonary venous connection: thirty-year trends. Circulation. 1996;94(suppl 2):12-6.

2. Raisher B, Grant J, Martin T, et al. Complete repair of total anomalous pulmonary venous connection in infancy. J Thorac Cardiovasc Surg. 1992;104:443-8.

3. Sano S, Brawn W, Mee R. Total anomalous pulmonary venous drainage. J Thorac Cardiovasc Surg. 1989;97:886-92.

4. Stark J. Anomalies of pulmonary venous return. World J Surg. 1985; 9:532-42.

5. Bogers A, Baak R, Lee P, et al. Early results and long-term follow-up after corrective surgery for total anomalous pulmonary venous return. Eur J Cardiothorac Surg. 1999;16:296-9.

6. Bellinger D, Wypij D, Kuban K, et al. Developmental and neurologic status of children at 4 years of age after heart surgery with hypothermic 
circulatory arrest or low-flow cardiopulmonary bypass. Circulation. 1999;100:526-32.

7. Bellinger D, Wypij D, duPlessis A, et al. Neurodevelopmental status at eight years in children with dextro-transposition of the great arteries: the Boston circulatory arrest trial. J Thorac Cardiovasc Surg. 2003; 126:1385-96.

8. Hovels-Gurich H, Seghaye M, Schnitker R, et al. Long-term neurodevelopmental outcomes in school-aged children after neonatal arterial switch operation. J Thorac Cardiovasc Surg. 2002;124:448-58.
9. Kirshbom P, Myung R, Gaynor J, et al. Preoperative pulmonary venous obstruction affects long-term outcome for survivors of total anomalous pulmonary venous connection repair. Ann Thorac Surg. 2002;74:1616-20.

10. Zhang J, Quan H, Ng J, Stepanavage M. Some statistical methods for multiple endpoints in clinical trials. Control Clin Trials. 1997;18:204-11.

11. Caldarone C, Najm H, Kadletz M, et al. Surgical management of total anomalous pulmonary venous drainage: impact of coexisting cardiac anomalies. Ann Thorac Surg. 1998;66:1521-6. 